\title{
UM ESTUDO DAS VARIÁVEIS QUE IMPACTAM A INOVAÇÃO EM EMPRESAS FAMILIARES E NÃO FAMILIARES: DISCUSSÃO COM FOCO NO PROGRAMA SEBRAE-ALI PERNAMBUCO
}

\author{
A STUDY OF VARIABLES THAT IMPACT ON INNOVATION IN \\ FAMILY AND NON-FAMILY ENTERPRISES: DISCUSSION WITH \\ A FOCUS ON SEBRAE-ALI PERNAMBUCO PROGRAM
}

Charles Ulises De Montreuil Carmona

RESUMO: O presente artigo busca analisar algumas metodologias de análise da inovação em empresas de pequeno porte e sua variação ao longo do ciclo (2016-2017) do Programa ALI. Assim, o trabalho contrapõe a abordagem do Radar da Inovação de Bauchmann e Destefani (2008) com os trabalhos de Murro (2013), De Massis et al (2015) e Nieto et al (2015). Para a realização do artigo foi utilizada uma amostra de 323 empresas correspondentes a 15 agentes locais de inovação que atuam em Pernambuco, utilizando as conclusões de artigos que utilizam a abordagem do radar da inovação (SEBRAE, 2015). Para análise das variáveis do programa no ciclo especificado foi utilizada a regressão logística. Os resultados mostram que houve um aumento discreto do grau de inovação das empresas apesar do aumento das dificuldades negociais como consequência da crise econômica do país durante o período analisado. Os dados da estatística descritiva mostram que as empresas se consolidam principalmente nos setores onde a formação dos empreendedores é cada vez mais especializada. Do mesmo modo, pode-se mencionar da relação entre a idade de fundação da empresa e os negócios do tipo familiar como aspectos relevantes nos resultados. Aspectos estratégicos como a alta autonomia decisional são atributos para o desenvolvimento e a permanência dos negócios familiares, principalmente o setor industrial.

PALAVRAS-CHAVE: Inovação; Empresas familiares e não familiares; Regressão logística; Programa SEBRAE-ALI; Pernambuco.

ABSTRACT: This article aims to analyze some methodologies of innovation analysis in small companies and their variation over the cycle (2016-2017) of SEBRAE ALI Program. Thus, the work contrasts Bauchmann and Destefani's Innovation Radar approach (2008) with the works of Murro (2013), De Massis et al (2015) and Nieto et al. (2015). For the accomplishment of the article, a sample of 323 companies corresponding to 15 local innovation agents that operate in Pernambuco, using the conclusions of articles using the innovation radar approach (SEBRAE, 2015) was used. For the analysis of the program variables in the specified cycle, logistic regression was used. The results show that there was a slight increase in the degree of innovation of the companies despite the increase in the difficulties of negotiations because of the economic crisis of the country during the analyzed period. Data from descriptive statistics show that firms consolidate mainly in sectors where entrepreneurship training is increasingly specialized. Likewise, the relationship between the age of foundation of the company and the businesses of the family type can be mentioned as relevant aspects in the results. Strategic aspects such as high decision-making autonomy are attributes for the development and permanence of family businesses, especially the industrial sector. 


\section{INTRODUÇÃO}

Atualmente, os investimentos realizados pelas empresas vêm enfrentando um conjunto de restrições de ordem financeira, operacional, de recursos, temporal, entre outras, que impactam no seu desempenho futuro (sAMANTRA et al., 2016; SCARPELLINI et al., 2016). Essa situação se potencializa quando se fala em projetos de inovação realizados por empresas, onde há a presença dos riscos subjacentes a própria inovação.

Por outro lado, em uma economia em que o risco, a insegurança e as constantes mudanças deixam de ser uma exceção para tornar-se regra, sob o ponto de vista organizacional, a inovação mostra-se como fonte originária de conhecimento e expertise, em busca de garantir a competitividade das empresas no seu setor de atuação (HAUSMAN e JOHNSTON, 2014; ARCHIBUGI et al., 2013), apesar dos riscos e incertezas decorrentes.

Portanto, as empresas devem inovar para sobreviver no mercado de forma ativa e adaptativa (BOWERS e KHORAKIAN, 2014), pois em momentos de recessão econômica, apesar das restrições financeiras, a inovação possibilita a conversão de crise em oportunidades viáveis ao promover o rompimento do padrão competitivo vigente, como já anunciavam Schumpeter (1985) e Christensen (1997), além de promover a excelência organizacional e agregação de valor para os negócios (YAM et al., 2004; VILLALONGA, 2004; CARVALHO et al., 2010).

As incertezas dos projetos inovadores têm sido estudados a partir de diferentes perspectivas e análises teóricas divergentes quanto à anatomia dos riscos dentro do contexto de valor informativo analisado, ou seja, os riscos são estudados sob várias dimensões.

O objetivo principal do artigo é identificar uma proposta de avaliação das variáveis determinantes da inovação de empresas familiares e não familiares, cujas estratégias foram realizadas por um conjunto de empresas do Programa Sebrae/Ali em Pernambuco.

\section{REFERENCIAL TEÓRICO}

No sentido econômico, a inovação é completa quando ocorre uma transação comercial envolvendo uma invenção e assim é capaz gerar riqueza (SCHUMPETER, 1988).

Nessa concepção, de acordo com a abordagem clássica de Schumpeter (1985), a inovação é a adoção de um novo método de produção, de criação e inserção de um novo produto no mercado, de uma nova forma de organização ou da conquista de um novo mercado. Ainda segundo o autor, mais precisamente, a inovação tecnológica, fonte de investigação do presente trabalho, pode ser observada quando a empresa implementa um novo produto no mercado ou quando adiciona um novo processo de produção na empresa, com o objetivo de acrescentar valor na atividade econômica (AUDRETSCH et al., 2002). É a partir da conceituação de Schumpeter que a inovação passa a ser analisada com maior propriedade sob a perspectiva do desenvolvimento econômico. 
Schumpeter (1985) destaca ainda o papel fundamental da inovação no ato de empreender e seu impacto no crescimento econômico (VALE, WILKINSON e AMÂNCIO, 2008). Havendo a distinção entre invenções (novas ideias e conceitos) e inovações (uma nova combinação de recursos produtivos), o autor caracteriza a inovação não apenas como um estado da arte para o qual incrementos e revoluções criativas são criadas, mas também a sua viabilidade para a materialização e a tangibilidade do seu resultado financeiro, conforme também advogam Bertazi e Salerno (2015).

Ainda segundo Schumpeter (1985), a inovação pode ser vista como um conjunto de funções evolutivas que alteram os métodos de produção, criando modernas formas de organização do trabalho e processos ao produzir novas mercadorias, possibilita a abertura de novos mercados mediante a criação de novos usos e consumos. $\mathrm{O}$ autor ressalta que nesse processo ocorrem "mudanças espontâneas e descontínuas nos canais de fluxo, perturbação do equilíbrio, que altera e desloca para sempre o estado de equilíbrio previamente existente" (SCHUMPETER, 1982, p. 47).

Como se percebe, o caminhar da inovação no sistema econômico não ocorre de maneira estática, desafia sempre as empresas e tecnologias vigentes rumo à destruição criativa, como Schumpeter (1982) denominou, ou seja, rumo a um novo patamar de desenvolvimento e de competitividade entre as empresas, que possui efeito cumulativo em todo o sistema capitalista. Segundo Bastos (2006), os diferentes ramos da atividade econômica apresentam dinâmicas setoriais próprias e para seu entendimento é necessário estudar os processos produtivos, tipos de produto e padrões de competição rumo à ruptura do padrão competitivo vigente.

A inovação pode ser vista também como um processo de oportunidade em torno de novas ideias e de colocá-las em prática (TIDD, BESSANT E PAVITT, 2008).

De uma forma mais abrangente, o manual de Oslo advoga que o conceito de inovação está relacionado com vários aspectos do ponto de vista empresarial, a saber: oferecimento de um novo bem onde um novo serviço é dirigido aos clientes; estabelecimento de um novo processo produtivo, bem como a otimização dos já existentes; adoção de uma nova estratégia de marketing para criação de um valor superior para a empresa; redirecionamento de como o negócio é conduzido e gerenciado através da utilização de estratégias que proporcionem vantagens competitivas para a empresa; as relações estabelecidas com o ambiente no qual a empresa está inserida (OCDE - Organisation for Economic Cooperation and Development, 2005). 


\section{TIPOS DE INOVAÇÃO}

Em pesquisas publicadas no Manual de Oslo (OCDE, 2005), as inovações podem ser enquadradas como de produto, de processo, de marketing ou organizacional. Segundo Henderson e Clark (1990), a inovação pode se mostrar na forma incremental, modular, arquitetônica ou radical. Esta última tipologia proposta está focada no impacto da inovação sobre a utilidade de uma arquitetura existente e sobre o conhecimento da empresa sobre os seus componentes. Por esse motivo, os autores consideram importante a ideia de design dominante e construção de conhecimento e competências das organizações a partir de tarefas recorrentes, por serem os principais conceitos para compreender o conhecimento, fonte de inovação (KLEMENT E YU, 2008).

A necessidade do entendimento dos tipos de inovação e suas diferentes características é necessário por exigir uma estrutura organizacional adequada para gerenciar o processo de inovação, bem como auxiliar os gestores na elaboração de suas estratégias e na alocação dos recursos (кIм et al., 2012).

As inovações do tipo incremental são aquelas que proporcionam melhorias significativas aos produtos, serviços ou processos já existentes. Esse tipo de inovação contribui para a melhoria contínua das capacidades centrais da empresa, fazendo com que atributos chaves dos produtos, serviços ou processos sejam cada vez mais aprimorados e direcionados para um melhor atendimento das necessidades dos clientes, possuindo um caráter de novidade ainda que não seja uma mudança abrupta, de ruptura, revolucionária, como a inovação radical (LIZARELLI E TOLEDO, 2015). A inovação incremental refere-se a pequenas alterações de tecnologias existentes em termos de design, função, preço, quantidade e características para atender às necessidades dos clientes existentes (KIM et al., 2012). Por outro lado, existem as inovações radicais que são definidas como a adoção de novas tecnologias para criar uma demanda ainda não reconhecida pelos clientes e mercados (JANSEN et al., 2006). Esse tipo de inovação apresenta características de desempenho sem precedentes ou características já conhecidas que transformem os mercados existentes ou criem novos mercados. Como se percebe, esse tipo de inovação está muito próximo dos conceitos da destruição criadora de Schumpeter e da inovação disruptiva de Christensen, pelo alto nível de incerteza e complexidade de condução de seus processos.

A inovação arquitetônica envolve a reconfiguração dos elementos que a empresa possui em sua produção, interligando-os de uma nova maneira, sem alteração do projeto dominante em desenvolvimento. Por outro lado, a inovação modular modifica o conceito do projeto dominante. A tipologia proposta por Henderson e Clark (1990) pode ser visualizada na Figura 1. 


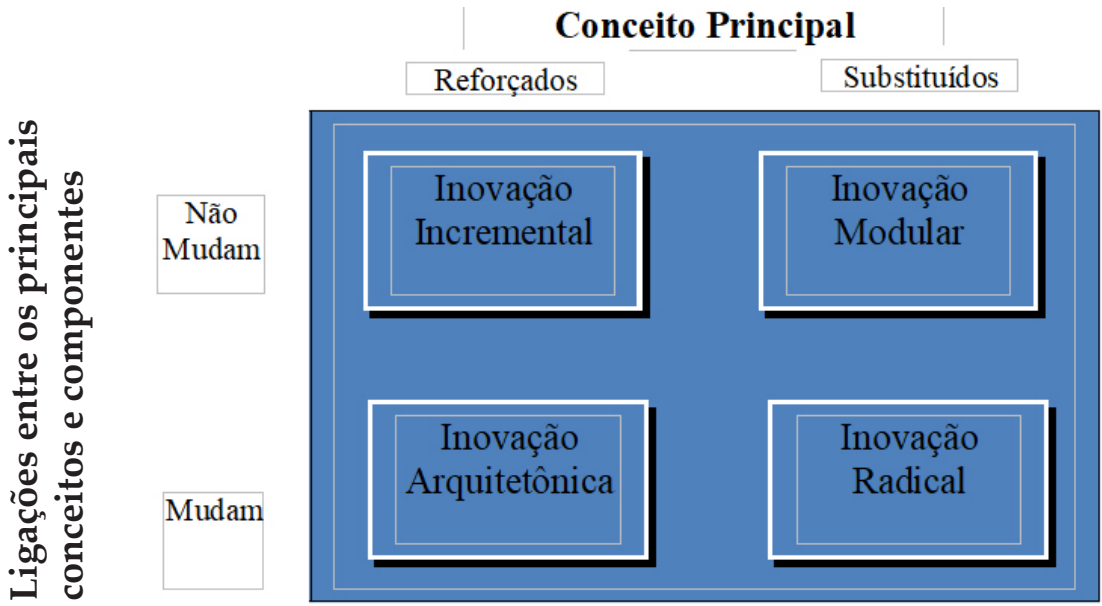

FONTE: Adaptado de Henderson e Clark (1990).

Por meio da Figura 1 percebe-se que está se caracteriza em um continuum,onde em seus extremos estão as inovações radicais e incrementais, ora rompendo com o conceito dos produtos, serviços ou processos vigentes, ora estabelecendo melhorias e reforçando os componentes desse conceito.

\subsection{ABORDAGEM DO RADAR DA INOVAÇÃO}

O Radar da Inovação, tal qual utilizado no programa ALI, é o resultado da combinação dos trabalhos de Sawhney et al (2006) e Bachmann e Destefani (2008).

Segundo Sawhney et al (2006), o Radar contém quatro dimensões principais: as ofertas que a empresa cria; os consumidores que ela serve; os processos que ela usa e os pontos de presença que ela usa para levar sua oferta ao mercado (sawhney et al, 2006, p. 4). Além dessas dimensões, os autores inseriram outras oito dimensões: Plataforma, Soluções, Experiência do Cliente, Agregação de valor, Organização, Cadeia de Fornecimento, Rede e Marca (sawhney et al, 2006, p. 4-7).

Bachmann e Destefani (2008) adaptaram o Radar de Sawhney et al (2006) para a realidade das MPEs brasileiras e inseriram uma nova dimensão, a Ambiência inovadora, "por entender que um clima organizacional propício à inovação é pré-requisito importante para uma empresa inovadora" (BACHMANN E DESTEFAnI, 2008, p. 9). Além desta, outras modificações foram feitas, a saber: 1 ) a metodologia passou a adotar "uma abordagem mais qualitativa que quantitativa, para respeitar a menor disponibilidade de informações que é típica das MPE" (BACHMANN E DESTEFAnI, 2008, p. 9); 2) a dimensão Experiência do Cliente adquiriu nova denominação, passando a chamar-se de Relacionamento e a dimensão Praça foi também chamada de Presença (BACHMAnN e DESTEFANI, 2008, p. 16).

Em consequência, tem-se que a partir de Bachmann e Destefani (2008), o Radar passou a conter 13 dimensões: Oferta, Plataforma, Marca, 
Clientes, Soluções, Agregação de Valor, Processos, Rede, Presença (Praça), Relacionamento, Cadeia de Fornecimento, Organização e Ambiência Inovadora. Juntas, as 13 Dimensões do Radar da Inovação totalizam, com as subclassificações, um conjunto de 42 itens.

Segundo Bachmann \& Associados (2014, p.27), as informações servem de referência para mudanças importantes criando oportunidades para a inovação. A Identificação de mercados analisa a busca proativa da empresa por novos mercados, que podem significar, de acordo com eles, grupos de clientes ou clientes com necessidades diferentes.

A Dimensão Relacionamento leva em conta tudo que o consumidor vê, ouve, sente ou experimenta de algum modo ao interagir com a empresa em todos os momentos (BACHMANN \& ASSOCIADOS, 2014, p. 30). Nessa dimensão, considera-se, portanto, a capacidade da empresa de manter relações com seus clientes, através de dois aspectos, representados por itens: Facilidades e Amenidades, e Informatização. Esses itens consideram aspectos do uso da tecnologia da informação para aumentar a qualidade da experiência com o cliente, quais sejam: redes sociais, desenvolvimento de site, entre outras novas tecnologias. A Tabela 1 apresenta a pontuação de cada grau de inovação conforme o nível específico em que se encontra a empresa.

TABELA 1 - Pontuação Grau de Inovação

\begin{tabular}{c|c} 
ESCORE & SITUAÇÃo \\
\hline 1 & Pouco ou nada inovadoras \\
\hline 3 & Inovações ocasionais \\
\hline 5 & Inovações sistêmicas \\
& FONTE: SEBRAE, 2015
\end{tabular}

\subsection{ABORDAGEM DOS FATORES DETERMINANTES DA "TENDÊNCIA"} À INOVAÇÃO POR PARTE DAS EMPRESAS: MURRO (2013)

O processo para a inovação não é tão linear quanto deveria, uma vez que as diferentes variáveis que se esperam que determinem e incentivem a inovação sejam tão numerosas que o problema das variáveis omitidas é muito susceptível de influenciar a interpretação de estudos empíricos. Os fatores que influenciam a inovação podem ser de natureza interna ou externa. O primeiro pode incluir características como idade, tamanho e ser membro de um determinado grupo; Características estratégicas, como a presença nos mercados estrangeiros; Características financeiras como a solidez de uma empresa e sua relação com o sistema bancário. Entre estes, pode haver o nível de competitividade do mercado, sua estrutura socioeconômica e a situação financeira de um país. Assim, esta metodologia apresenta (ver Tabela 2) uma série de variáveis consideradas relevantes para a inovação como estratégia de agregação de valor para as empresas. 
Tabela 2. Variáveis Determinantes da abordagem de tendência à Inovação

\begin{tabular}{|c|c|}
\hline VARIÁVEIS & DEFINIÇÕES \\
\hline \multicolumn{2}{|c|}{ Variáveis dependentes } \\
\hline Innovat & Binária, se a empresa relata inovações significativas \\
\hline Innoprod & Binária, se a empresa relata inovações de produto \\
\hline Innoproc & $\begin{array}{l}\text { Binária, se a empresa relata inovações de processo } \\
\text { ou organizacionais }\end{array}$ \\
\hline \multicolumn{2}{|c|}{ Variáveis de Controle } \\
\hline Tamanho & Logaritmo do número de funcionários \\
\hline Rede & Binária. Toma o valor 1 se faz parte de uma rede \\
\hline Norte & $\begin{array}{l}\text { Binária. Toma o valor } 1 \text { se está localizada na mata } \\
\text { norte }\end{array}$ \\
\hline Capital & $\begin{array}{l}\text { Binária. Toma o valor } 1 \text { se está localizada na região } \\
\text { metropolitana do Recife }\end{array}$ \\
\hline Sul & $\begin{array}{l}\text { Binária. Toma o valor } 1 \text { se está localizada na mata } \\
\text { sul. }\end{array}$ \\
\hline Interior & $\begin{array}{l}\text { Binária. Toma o valor } 1 \text { se está localizada no } \\
\text { Interior. }\end{array}$ \\
\hline Educ1 & $\begin{array}{l}\text { Percentual de empregados com ensino fundamental } \\
\text { completo. }\end{array}$ \\
\hline Educ2 & $\begin{array}{l}\text { Percentual de empregados com ensino médio } \\
\text { completo. }\end{array}$ \\
\hline Idade & $\begin{array}{l}\text { Logaritmo do número de anos de fundação do } \\
\text { negócio. }\end{array}$ \\
\hline Growth & $\begin{array}{l}\text { Média aritmética da taxa de crescimento da região } \\
\text { onde a empresa está localizada. }\end{array}$ \\
\hline Trad & $\begin{array}{l}\text { Binária. Toma o valor } 1 \text { se a empresa atua num setor } \\
\text { muito tradicional. }\end{array}$ \\
\hline Stand & $\begin{array}{l}\text { Binária. Toma o valor } 1 \text { se a empresa atua num setor } \\
\text { muito com processos padronizados. }\end{array}$ \\
\hline HTech & $\begin{array}{l}\text { Binária. Toma o valor } 1 \text { se a empresa atua num setor } \\
\text { com alto grau de Tecnologia. }\end{array}$ \\
\hline
\end{tabular}

FONTE: Adaptado de Murro et al (2013)

Portanto, a metodologia anterior foca na avaliação do nível de desempenho da empresa.

ABORDAGEM CONSIDERANDO A SITUAÇÃO DE EMPRESAS

FAMILIARES E NÃO FAMILIARES: DE MASSIS, FRATINI, PIZZURNO

E CASSIA (2015) E NIETO, SANTAMARIA E FERNANDEZ (2015)

Como as empresas familiares gerenciam a inovação de produtos continua a ser um tópico esquecido na pesquisa comercial existente. Isso ocorre apesar do fato de que as empresas familiares desempenham um papel crucial em todas as economias, e muitas vezes usam a inovação tecnológica para estimular sua vantagem competitiva. Através da visão baseada em recursos da empresa, bem como das teorias de agência, administração e comportamento e usando evidências empíricas reunidas através de um estudo de caso múltiplo, o artigo estuda como e por que a anatomia do processo de inovação do produto difere entre empresas familiares e não familiares. A Figura 2 apresenta mostra a análise realizada por De Massis at al (2015) partindo da base de que as empresas familiares diferem das não familiares, no que se refere às estratégias de inovação de produtos, o risco assumido e à organização no próprio processo de inovação. 
FIGURA 2 - Aspectos-chaves no Processo de Inovação de empresas familiares e não familiares

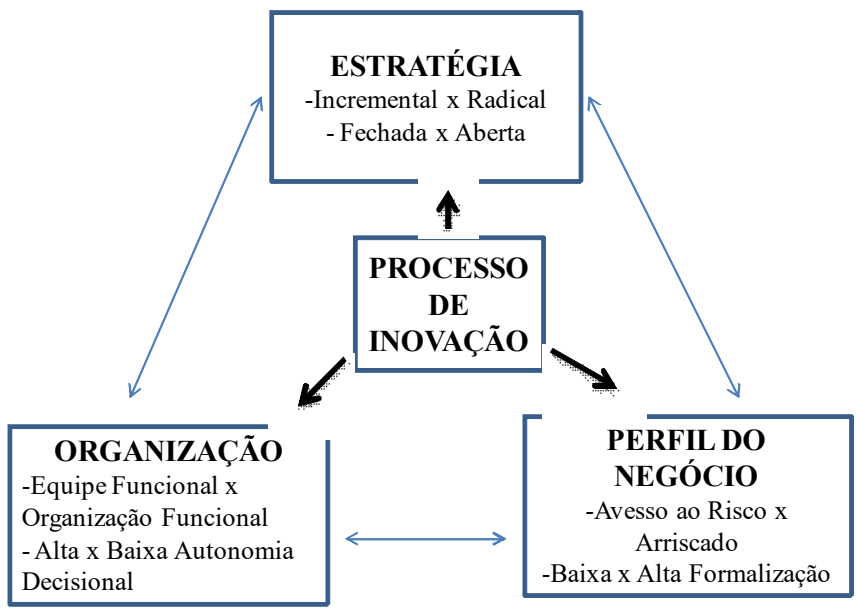

FONTE: Adaptado de De Massis et al (2015)

A Figura 2 tipifica as principais características das empresas familiares em relação com o processo de inovação, com estratégias do negócio, o tipo de organização dada a autonomia e funcionalidade e finalmente, o perfil do negocio em relação à aversão ao risco e formalização definidas pelos proprietários do negócio.

Por outro lado, a pesquisa realizada por Nieto et al (2015) fundamenta as diferenças entre as empresas familiares e não familiares por meio de uma relação funcional cujas principais variáveis estão relacionadas na Tabela 3, a seguir.

TABELA 3 - Variáveis Determinantes da abordagem das empresas familiares

\begin{tabular}{|c|c|}
\hline VARIÁVEIS & DEFINIÇÕES \\
\hline \multicolumn{2}{|c|}{ Variáveis dependentes } \\
\hline EInova1 & $\begin{array}{l}\text { Esforço de Inovação. Relação entre investimento entre P\&D } \\
\text { (ou atividades relacionadas com a inovação) dividido pelo } \\
\text { faturamento total. }\end{array}$ \\
\hline EInova2 & $\begin{array}{l}\text { Binária, toma o valor } 1 \text { se a empresa relata inovações de pro- } \\
\text { cesso ou organizacionais. Também pode tomar o valor } 1 \text { quan- } \\
\text { do analisamos as inovações radicais vs. Incrementais. }\end{array}$ \\
\hline \multicolumn{2}{|c|}{ Variáveis de Controle } \\
\hline Fam & Binária. Toma o valor 1 em caso de empresas familiares. \\
\hline Tamanho & Logaritmo do número de funcionários. \\
\hline Mksh & O market share é utilizado como proxy do poder de mercado. \\
\hline Idade & Logaritmo da idade da empresa desde a fundação da mesma. \\
\hline Alav & $\begin{array}{l}\text { Alavancagem. Se estima dividindo o capital de terceiros pelo } \\
\text { capital próprio. }\end{array}$ \\
\hline Conclien & $\begin{array}{l}\text { Concentração de clientes. Calcula-se dividindo a soma das } \\
\text { vendas dos três principais clientes sobre as vendas totais. }\end{array}$ \\
\hline Confor & $\begin{array}{l}\text { Concentração de fornecedores. Calcula-se dividindo a soma } \\
\text { das compras dos três principais fornecedores sobre as com- } \\
\text { pras totais. }\end{array}$ \\
\hline Aprop & $\begin{array}{l}\text { "Apropriabilidade" que pode ser representada por uma } \\
\text { variável binária com valor } 1 \text { quando o setor apresenta um } \\
\text { bom sistema de proteção que promove as atividades de in- } \\
\text { ovação. }\end{array}$ \\
\hline
\end{tabular}

FONTE: Adaptado de Nieto et al (2015) 
A Apropriabilidade é importante, pois existem setores mais inovadores do que outros, e pode ser estimada dividindo o número de patentes geradas pela empresa em relação ao total de patentes do setor. Contudo, esse cálculo às vezes é difícil de ser realizado.

\section{METODOLOGIA}

A presente seção aborda as seguintes etapas metodológicas: o lócus de investigação da pesquisa, o tipo e a coleta de dados. O método de pesquisa adotado neste trabalho é o estudo de multicaso, com abordagem qualitativa e quantitativa, que permite o melhor conhecimento dos processos e das relações estabelecidas entre os elementos existentes (DENCKER, 2008). A análise Logit ou regressão logística consiste em uma técnica utilizada na separação de dois grupos (familiares e não familiares), que visa obter a probabilidade de que uma observação pertença a um conjunto determinado, em função do comportamento das variáveis independentes (Hair et al, 2005). Ela é comumente utilizada em para análise de dados com resposta binária ou dicotômica e consiste em relacionar, através de um modelo, a variável resposta (variável dependente binária) com fatores que influenciam ou não a probabilidade de ocorrência de determinado evento (variáveis independentes). Uma característica importante da regressão logística é a flexibilidade em analisar variáveis cuja normalidade não é satisfeita. Para verificar os resultados faremos uso dos softwares Excel e SPSS.

O presente artigo analisará os dados tomando como base os resultados dos agentes locais de inovação de acordo com a abordagem do Radar da Inovação, considerando as observações da amostra selecionada.

\subsection{COLETA DE DADOS}

A amostra selecionada corresponde aos resultados de 15 agentes locais de inovação do ciclo 2 (2016-2017) que desenvolveram seus trabalhos de análise, sob a minha orientação, num total de 323 empresas. Por fazer parte do Programa ALI, as empresas correspondem aos mais diversos setores de serviços e varejo pernambucano.

\section{ANÁLISE DOS RESULTADOS}

De acordo com os dados analisados pelos ALIs podemos fazer uma descrição das observações sobre o aumento no nível de inovação utilizando o Radar da Inovação com as dimensões aplicadas para o aumento do grau de inovação (Ver Tabela 4). 
TABELA 4 - Dimensões do radar mais utilizadas

\begin{tabular}{l|c}
\multicolumn{1}{c|}{ DIMENSÃO } & $\%$ CASOS \\
\hline Relacionamentos & $28 \%$ \\
\hline Processo & $16 \%$ \\
\hline Marca & $8 \%$ \\
\hline Clientes & $8 \%$ \\
\hline Rede & $8 \%$ \\
\hline Ambiência Inovadora & $16 \%$ \\
\hline Agregação de Valor & $4 \%$ \\
\hline Presença & $4 \%$ \\
\hline Organização & $4 \%$ \\
\hline Oferta & $4 \%$ \\
\hline
\end{tabular}

FONTE: Os autores (2018)

A tabela 4 mostra que a dimensão relacionamento foi a mais utilizada tendo em vista a crise financeira que afetou a região e que determinou variações mínimas dos preços dos produtos, mas uma aproximação maior com os clientes, padronização/simplificação de processos e mais foco para atingir o universo de interesse dos clientes. Para avaliar a evolução das empresas analisadas, a Tabela 5 mostra a variação do grau de inovação global.

TABELA 5 - Variação do grau de inovação em valores médios (323 empresas)

\begin{tabular}{c|c|c|c|} 
GRAU INOVAÇÃO GLOBAL & $\mathrm{R}(0)$ & $\mathrm{R}(1)$ & VARIAÇÃO \\
& 2,071 & 2,216 & $7 \%$
\end{tabular}
FONTE: Os autores (2018)

Um aumento médio de $7 \%$ no grau de inovação do nível R(o) para $R(\mathrm{I})$ é bastante representativo considerando o período de turbulências enfrentado pelos empresários, tendo como objetivo a manutenção das margens e o retorno financeiro.

TABELA 6 - Análise das empresas inovadoras familiares e não familiares

\begin{tabular}{c|c|c|c|c|c} 
CONSTANTE & IDADE & TAMANHO & COMÉRCIO & SERVIÇOS & INDÚSTRIA \\
\hline$-0,061$ & $\begin{array}{c}0,121^{* *} \\
\left(\begin{array}{c}* * * \\
(* *)\end{array}\right)\end{array}$ & $\left.\begin{array}{c}-0,004 \\
(*)\end{array}\right)$ de significância & 0,001 & $0,233^{* *}$ & $0,499^{* * *}$ \\
& & & \\
\end{tabular}

FONTE: Elaborado pelos autores (2018)

Os resultados da tabela anterior evidenciam uma associação dos negócios dos setores de Indústria (altamente significante) e Serviços (significância relativa) relacionados com as empresas familiares. Do mesmo modo, pode-se mencionar da relação entre a idade de fundação da empresa e os negócios do tipo familiar como aspectos relevantes nos resultados. Aspectos estratégicos como a alta autonomia decisional são atributos para o desenvolvimento e a permanência dos negócios familiares nesses setores, principalmente o setor industrial. 
Na avaliação qualitativa da inovação podem-se destacar as seguintes ações que foram implementadas:

- Em geral, necessidade de aumentar a visibilidade dos negócios interagindo mais com as mídias sociais, e em particular as mais conhecidas;

- Melhorar os canais de comunicação com os clientes;

- Melhorar o processo de pós-venda por produto/serviço com os clientes;

- Sistematizar os processos através da implementação de protocolos de execução;

- Na dimensão Relacionamento a atividade de "informatização" tem se tornado mais difícil de implementar.

$\mathrm{Na}$ avaliação qualitativa da inovação também podem-se destacar as seguintes ações que se sugerem implementar:

- Sistematizar o processo de captação de novos clientes para aumentar a participação de mercado do negócio;

- Melhorar o processo de descarte de materiais e subprodutos com o objetivo de reaproveitamento de matéria prima e, por conseguinte, na direção da redução de custos e ações sustentáveis.

- Estruturar um processo para fidelização dos clientes, elaborando um plano de fidelização e possibilitando o aumento da taxa de retorno dos mesmos através de vantagens oferecidas pela empresa.

\section{CONCLUSÃO}

Os resultados mostram que houve um aumento discreto do grau de inovação global das empresas que compõem a amostra objeto de estudo. Isso significa que as empresas obtiveram sucesso na implementação de ações inovadoras, apesar da crise econômica que atravessa o país nos últimos três anos.

Um ponto importante a ser destacado é o relato dos empreendedores que buscam continuamente melhorias no âmbito empresarial e aumento da competitividade, e que atingiram os objetivos com sucesso. O processo de inovação não está associado necessariamente ao aumento de custos, pode ser obtido por meio de melhorias nas rotinas organizacionais e nos processos produtivos.

Assim, a abordagem do Radar da Inovação se mostrou de utilidade, justamente por representar uma ferramenta qualitativa quantitativa de análise. 
Os dados da estatística descritiva mostram que as empresas se consolidam principalmente nos setores onde a formação dos empreendedores é cada vez mais especializada. Não apenas onde o mercado é maior, o acesso às informações e tecnologia facilita o crescimento empresarial e a manutenção do retorno financeiro. Essas orientações para estudos futuros beneficiarão intensamente a pesquisa sobre gestão de negócios e inovação de negócios famíliares, pois considera teorica e empiricamente como o envolvimento da família na propriedade, governança e gestão afeta a inovação.

\section{REFERÊNCIAS}

AUDRETSCH, D.B.; BOZEMAN, B.; COMBS, K. L.; FELDMAN, M.; LINK, A. N.; SIEGEL, D. S.; STEPHAN, P.; TASSEY, G.; WESSNER, C. The economics of science and Technology. Journal of Technology Transfer. v. 27, n. 2, p. 155-203, 2002.

BACHMANN, D. L.; DESTEFANI, J. H. Metodologia para estimar o grau das inovações nas MPE. Curitiba: SEBRAE, 2008.

BACHMANN, D. Guia para a Inovação: Instrumento para a melhoria das dimensões da inovação. Curitiba, PR, 2010.

BASTOS, T. P. Gestão da inovação: a economia da tecnologia no Brasil. Rio de Janeiro: Elsevier, 2006.

BERTAZI, L. E. A.; SALERNO, M. S. Evolução e panorama da pesquisa em indicadores de inovação. In: Simpósio de engenharia de produção, 15, 2015, São Paulo, Anais... São Paulo: 2015, p. 1-15.

CARVALHO, F. M.; KAYKO, E. K.; MARTIN, D. M. L. Tangibilidade e intangibilidade na determinação do desempenho persistente de firmas brasileiras.

Revista de Administração Contemporânea, Curitiba, v. 14, n. 5, p. 871-889, 2010.

CHRISTENSEN, C. M.The Innovator's Dilemma, Harvard Business School Press, 1997.

DENCKER, A. Pesquisa Empírica em Ciências

Humanas. São Paulo: Editora Futura, 2008.

DE MASSIS, A.; FRATTINI, F.; PIZZURNO, E.; CASSIA, L. Product Innovation in Family versus Nonfamily Firms: An Exploratory Analysis. Journal of Small Business Management 2015 53(1), pp. 1-36.

HAIR JR., J.F.; BLACK, W.; TATHAM, R.; ANDERSON, R.E. Análise multivariada de dados. 5.ed. Porto Alegre: Bookman, 2005. 
HAUSMAN, A.; JOHNSTON, W. J. The role of innovation in driving the economy: Lessons from the global financial crisis. Journal of Business Research, v. 67, n. 1, p. 2720-2726, 2014.

HENDERSON, R.M.; CLARK, K.B. Architectural innovation: the reconfiguration of existing product technologies and the failure of established firms.

Administrative Science Quarterly, v. 35, p. 9-30, 1990.

JANSEN, J.; VAN DEN BOSCH, F.A.J.; VOLBERDA, H. W. Exploratory innovation, exploitative innovation, and performance: effects of organizational antecedentsand environmental moderators.

Management Science. n. 52, v.11, p. 1661-1674, 2006.

KIM, D. Y.; KUMAR, V.; KUMAR, U.Relationship between quality management practices and innovation. Journal of Operations Management, v. 30, n. 4, p. 295-315, 2012.

KLEMENT, C. F. F.; YU, A. S. O. Influências da tecnologia para a inovação em serviços. Revista de Administração da UFSM, v. 1, n. 1, art. 7, p. 101-115, 2008.

LIZARELLI, F. L.; TOLEDO, J. C. Identificação de relações entre melhoria contínua e inovação de produtos e processos por meio de revisão bibliográfica sistemática. Gestão \& Produção, São Carlos, v. 22, n. 3, p. 590-610, 2015.

MURRO, PIERLUIGI. Determinants of innovation: what is the role of risk?. The Manchester

School, Vol. 81 No. 3 293-323, June 2013.

NIETO, M. J.; SANTAMARIA, L.; FERNANDEZ, Z. Understanding the Innovation Behavior of Family Firms. Journal of Small Business Management, 2015 53(2), pp. 382-399.

OCDE. Manual de Oslo: Diretrizes para a coleta e interpretação de dados sobre Inovação. 3 ed., Tradução FINEP, 2005.

SAMANTRA, C.; DATTA, S.; MAHAPATRA, S. S. Interpretive structural modelling of critical risk factors insoftware engineering project. Benchmarking:An InternationalJournal, v. 23, n. 1, p. 2-24, 2016.

SAWHNEY. M.; WOLCOTT, R.; ARRONIZ, I. As 12 dimensões da Inovação. MIT Sloan Management Review, Cambridge, p. 75-8, 2006. 
SCARPELLINI, S.; VALERO-GIL, J.; PORTILLO-

TARRAGONA, P. The "economic-finance interface" for eco-innovation projects. International Journal of Project Management, v. 34, p. 1012-1025, 2016.

SCHUMPETER, J. A. (1947). The theoretical problems of economic growth in essays on entrepreneurs, business cycles and on the evolution of capitalism. Transaction Publishers, 1977.

SCHUMPETER, J. Business Cycles. New York Toronto London: McGraw-Hill Book Company, 1939.

SCHUMPETER, J. A. 1889-1950. Teoria do desenvolvimento econômico: uma investigação sobre lucros, capital, credito, juro e o ciclo econômico. 2. ed. São Paulo: Nova Cultural, 1985.

SEBRAE. Serviço Brasileiro de Apoio às Micro e Pequenas Empresas. Participação das Micro e Pequenas Empresas na Economia Brasileira relatório executivo, 2015.

TIDD, J.; BESSANT, J.; PAVITT, K. Gestão da inovação. Porto Alegre: Bookman, 2008.

VILLALONGA, B. Intangible resources, Tobin's Q, and sustainability of performance differences. Journal of Economic Behavior \& Organization, v. 54, n. 2, p. 205-230, 2004.

YAM, R. C. M.; GUAN, J. C.; PUN, K. F.; TANG, E. P. Y.An audit of technological innovation capabilities in chinese firms: some empirical findings in Beijing, China. Research Policy, v. 33, N. 8, p. 1123-1140. 2004. 